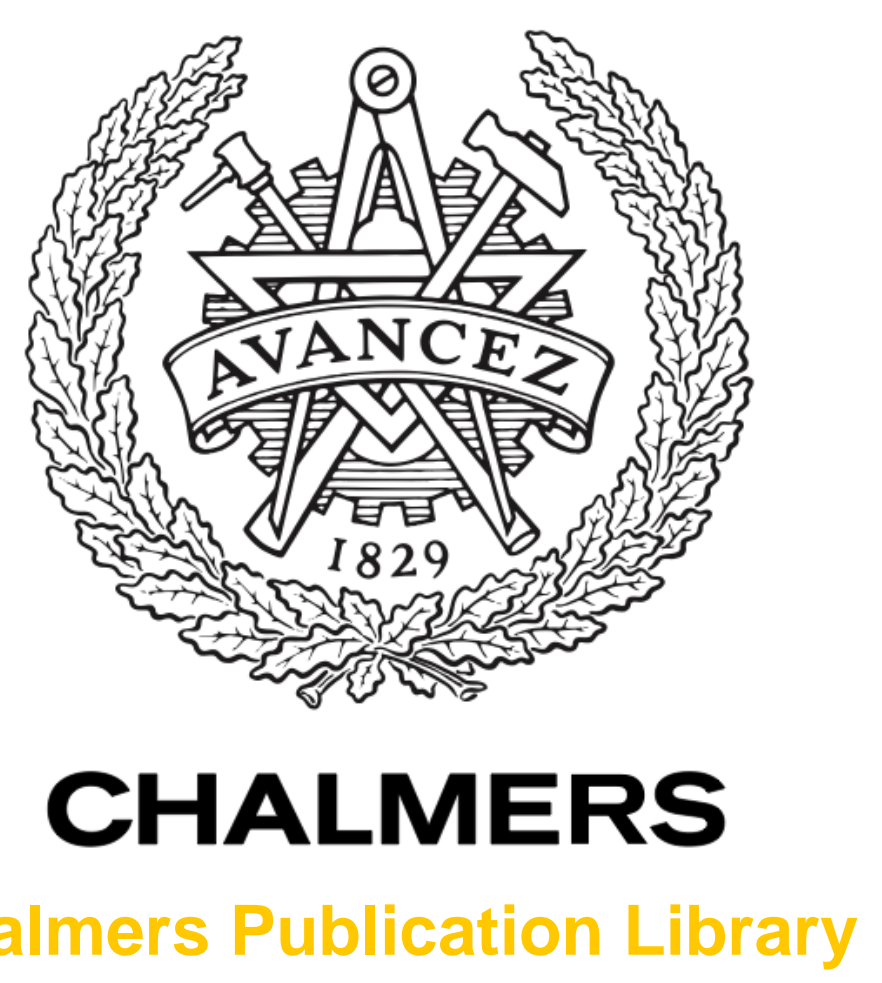

Chalmers Publication Library

\title{
The Self-Grounded Bow-Tie Antenna
}

This document has been downloaded from Chalmers Publication Library (CPL). It is the author's version of a work that was accepted for publication in:

2011 IEEE International Symposium on Antennas and Propagation, Spokane, USA, 3-8 July, 2011 (ISSN: 1522-3965)

Citation for the published paper:

Yang, J. ; Kishk, A. (2011) "The Self-Grounded Bow-Tie Antenna". 2011 IEEE International Symposium on Antennas and Propagation, Spokane, USA, 3-8 July, 2011 pp. 1452-1455.

http://dx.doi.org/10.1109/APS.2011.5996567

Downloaded from: http://publications.lib.chalmers.se/publication/143609

Notice: Changes introduced as a result of publishing processes such as copy-editing and formatting may not be reflected in this document. For a definitive version of this work, please refer to the published source. Please note that access to the published version might require a subscription. 


\section{The Self-Grounded Bow-Tie Antenna}

\author{
Jian Yang \\ Dept. of Signals and Systems \\ Chalmers University of Technology \\ Gothenburg, Sweden \\ jian.yang@chalmers.se
}

\author{
Ahmed Kishk \\ Department of Electrical Engineering \\ University of Mississippi \\ MS 38677 USA \\ ahmed@olemiss.edu
}

\begin{abstract}
The self-grounded Bow-Tie is a new type ultrawide band (UWB) small antenna. It is compact, simple and has low profile and directional UWB radiation characteristics. Simulations and measurements have shown its ultra-wideband performance, such as about $-10 \mathrm{~dB}$ reflection coefficient and stable radiation patterns over a frequency range of 2 - 15 $\mathrm{GHz}$, and good time-domain impulse response. Penetration ability of the antenna through a concrete wall has been also tested. Compared to a Vivaldi antenna, the test shows the superiority of the self-grounded Bow-Tie to the Vivaldi.
\end{abstract}

Index Terms-self-grounded Bow-Tie, UWB antenna, small antenna, directional radiation, impulse response.

\section{INTRODUCTION}

In many ultra-wide band (UWB) applications, small directional UWB antennas with low-profile in radiation direction have many advantages, such as low interference level, high penetration ability and compact geometrical configuration. However, few such UWB antennas have been reported.

Most of small UWB antennas are omnidirectional in radiation characteristics, for example, Bow-tie dipole [1]-[2]. The most common directional UWB antennas are of the travelingwave structure type, such as Vivaldi antennas [3] and Lindgren horn [4]. However, this type of UWB antennas have highprofile in the radiation direction which is a drawback in many UWB applications. Recently dielectric resonator antenna has also been designed for UWB applications [5].

This paper presents a new type UWB antenna - the selfgrounded Bow-tie. It is small in size with low-profile. It has ultra-wide band performance with about $-10 \mathrm{~dB}$ reflection coefficient, directional radiation characteristics and stable radiation beams for the frequency range of $2-15 \mathrm{GHz}$, and good time-domain impulse response.

\section{Geometrical Configuration of Self-Grounded BOW-TIE}

The geometrical configuration of the self-grounded BowTie antenna can be described as follows. An tilted infinite Bow-tie dipole is located above a ground plane, which is referred as to a seagull-over-sea configuration, see Fig. 1. Due to this configuration, the antenna is considered as a frequency independent one with directional radiation characteristics. However, for practicality, the structure should be truncated. The truncation is done by connecting the radiating element to the ground at the outer end of the antenna. Therefore, the

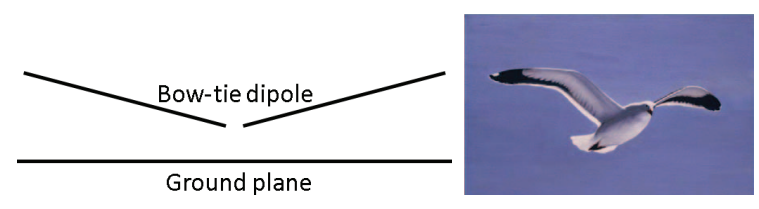

Fig. 1. Seagull-over-sea configuration of Bow-tie.

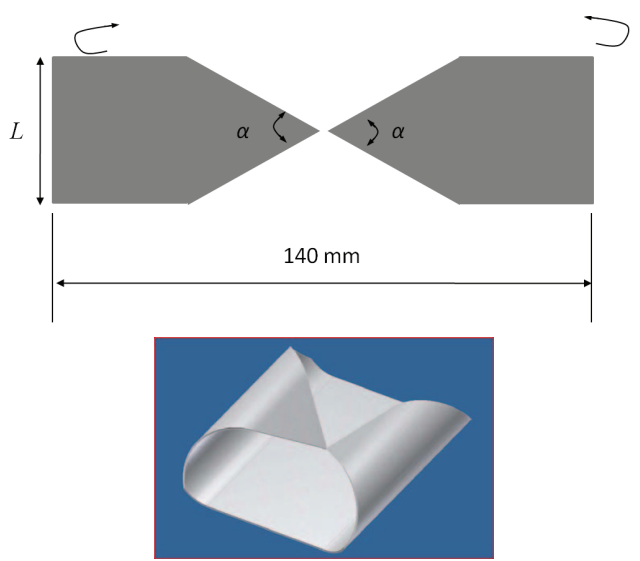

Fig. 2. Conversion from Bow-tie antenna to self-grounded Bow-tie antenna.

new antenna is referred to as the self-grounded Bow-Tie. This truncation method is partly inspired by our other UWB antenna research activities [6]-[12]. Fig. 2 shows the procedure of the conversion from a Bow-Tie to the self-grounded Bow-Tie.

\section{Simulations And Measurements}

A hardware of the self-grounded Bow-Tie antenna with the extended angle of $\alpha=60^{\circ}$ has been manufactured, with a size of $54 \times 58 \times 24 \mathrm{~mm}^{3}$, see Fig. 3. All simulation results in the paper are obtained by using CST MS [13].

Fig. 4 shows the measured and simulated reflection coefficient of the self-grounded Bow-Tie antenna. It can be observed that the reflection coefficient is below $-10 \mathrm{~dB}$ over the most part of 2 - $15 \mathrm{GHz}$, and below $-7 \mathrm{~dB}$ over the whole band. We believe that this performance can be improved to be below -10 $\mathrm{dB}$ over the whole band of $2-15 \mathrm{GHz}$ and even over a wider band width by employing some optimization schemes.

The self-grounded Bow-tie is a small UWB antenna. According to [14], the theoretical limitation of small antennas 


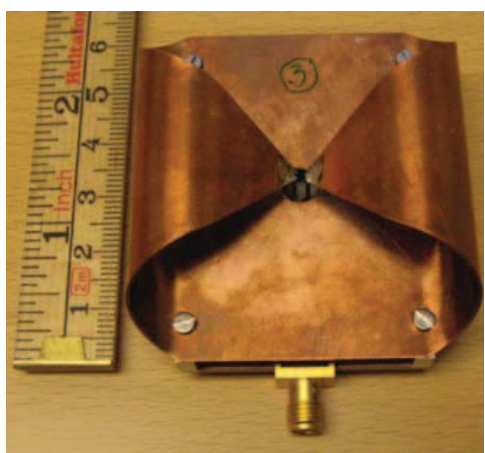

Fig. 3. Measured and simulated reflection coefficient of the self-grounded Bow-tie antenna with the balun.

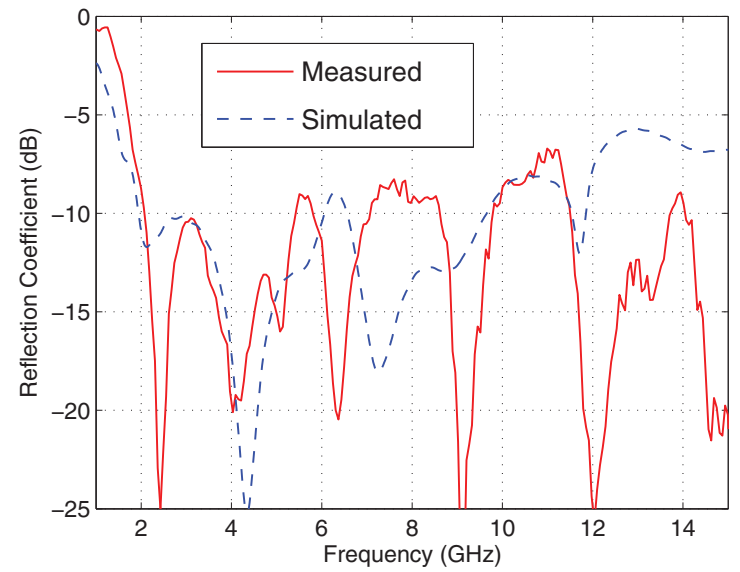

Fig. 4. Measured and simulated reflection coefficient of the self-grounded Bow-tie antenna with the feeding balun.

by the cut-off for gradual transition antennas presented is $\lambda_{\text {cutoff }}=2 \pi a$, where $a$ is the radius of the smallest sphere surrounding the antenna and $\lambda_{\text {cutoff }}$ is the wavelength of the lowest operating frequency for the antenna. For the selfgrounded Bow-Tie in the paper, we have $a=0.7 \cdot 58 \mathrm{~mm}=$ $40 \mathrm{~mm}$. Therefore, $\lambda_{\text {cutoff }}=6.28 \cdot 40 \mathrm{~mm}=240 \mathrm{~mm}$, i.e. $f_{\text {cutoff }}=300 / 240 \mathrm{~mm}=1.25 \mathrm{GHz}$, which matches pretty well the result in Fig. 4. Thus, the self-grounded Bow-tie is very close to the theoretical size limitation for small antennas.

Fig. 5 shows the simulated 3D radiation patterns. From the figure, we can see that the main radiation direction is along the normal of the ground plane, except around $7 \mathrm{GHz}$. The simulated and measured co- and cross-polar radiation patterns in $\varphi=45^{\circ}$ is shown in Fig. 6. The measurement confirms that the antenna has the directive radiation characteristics over the whole band of 2 - $15 \mathrm{GHz}$. The agreement between the simulated and the measured radiation patterns is good in a general sense. The reason for some difference for the detailed beam shapes we believe is the effect of the connectors and the cable used in the pattern measurement.

Fig. 7 shows the measured and simulated directivity of the

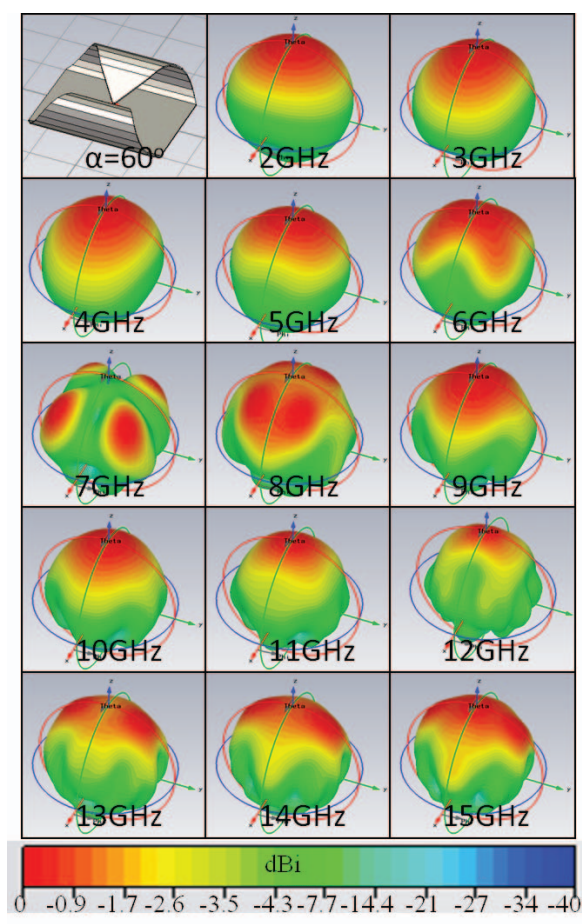

Fig. 5. Measured and simulated reflection coefficient of the self-grounded Bow-tie antenna with the feeding balun.

self-grounded Bow-tie antenna. The directivity is about 5 - 8 $\mathrm{dBi}$ over the frequency band of $2-15 \mathrm{GHz}$, which means that this antenna has quite stable radiation patterns compared to other UWB antennas, for example, open boundary quadridged horn [4], where the directivity varies from 6 to $13 \mathrm{dBi}$ over 2 - $15 \mathrm{GHz}$.

\section{TIme-Domain Response Measurement And Penetration Ability Test}

The time-domain response of the self-grounded Bow-Tie antenna was measured by an UWB radar system consisting of one NOVELDA UWB Radar kit R2A chip [15] and two self-grounded Bow-Ties (one is for transmitting, the other for receiving), see Fig. 8. Fig. 9 shows the time-response when the two self-grounded Bow-Ties are separated by a distance of $250 \mathrm{~mm}$ in face-to-face configuration. From the figure, we have the time-response measures of $\tau_{F W H M}=134 \mathrm{ps}$ and $\tau_{r=0.22}=260 \mathrm{ps}$. Please refer to [16] for the definitions of $\tau_{F W H M}$ and $\tau_{r=0.22}$.

The penetration ability has been also tested by using the same UWB radar system. For comparison, two Vivaldi antenna (size of $144 \times 135 \times 2 \mathrm{~mm}^{3}$, about 6 time larger than the self-grounded Bow-Tie in area, see Fig. 8) were also used in the test. The obstacle was a concrete wall of 515 millimeter thick and the targets were a metal cookie jar of $213 \mathrm{~mm}$ in diameter and a human being behind the wall, see Fig. 10. the transmitting and receiving antennas are in the same side of the wall. 


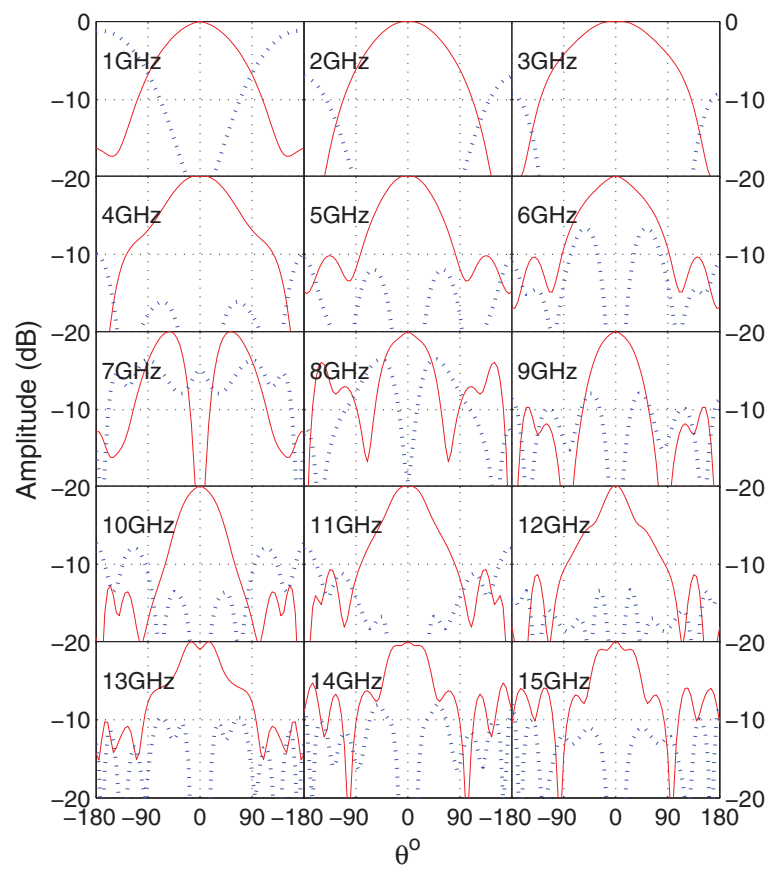

(a) Simulated

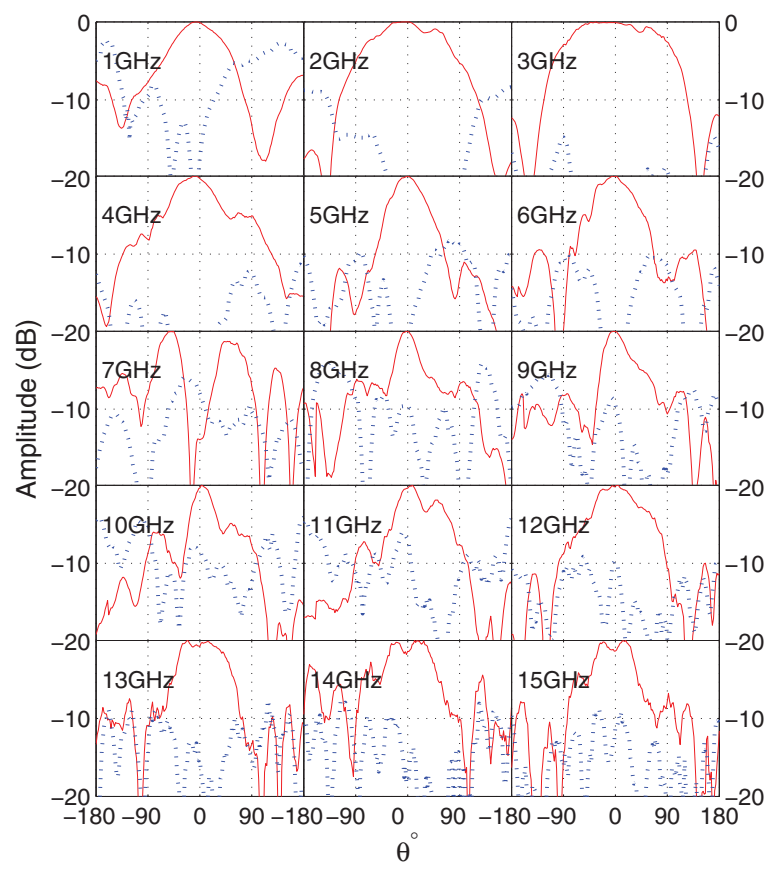

(b) Measured

Fig. 6. Co-(solid line) and cross-(dashed) polar radiation patterns in $\varphi=45^{\circ}$ of the self-grounded Bow-tie antenna with the balun.

The pulse signal is transmitted via the transmitting antenna, penetrates through the wall, hits the target, reflected, penetrates again through the wall and finally received by the receiving

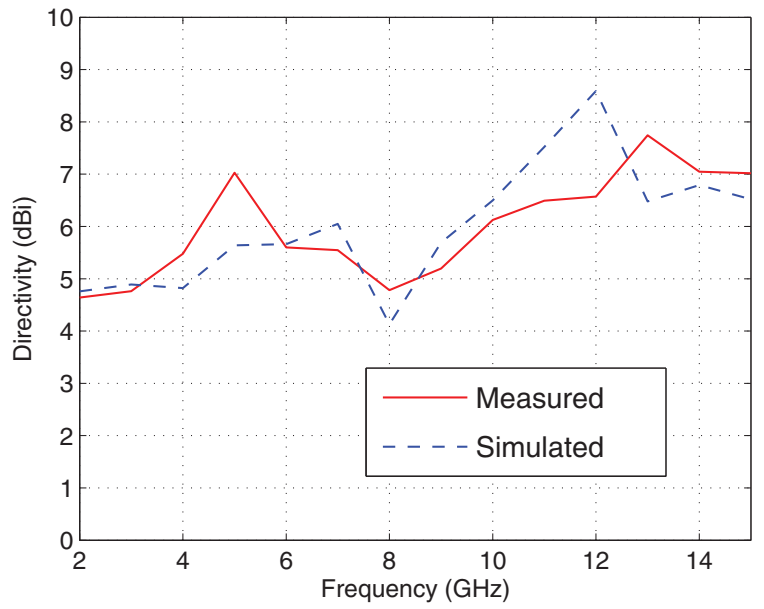

Fig. 7. Simulated directivity of the self-grounded Bow-Tie antennas of $60^{\circ}$ extended angle using CST

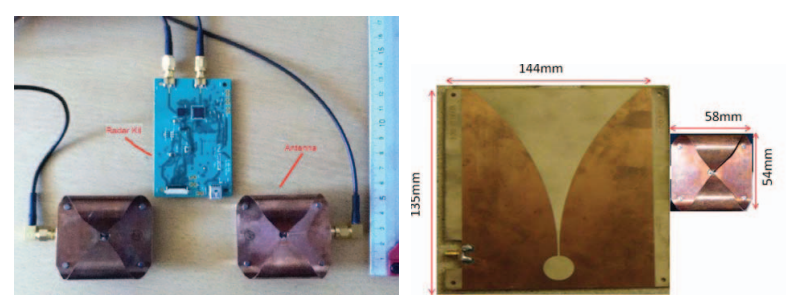

Fig. 8. The UWB radar system consisting of one Novelda radar chip and two self-grounded Bow-Ties (left), and size comparison of the Vivalde and the self-grounded Bow-Tie (right) used in the penetration test.

antenna. The results are shown in Fig. 11 and Fig. 12. From the figures, it is observed that the self-grounded Bow-Tie system has $7 \mathrm{~dB}$ and $5 \mathrm{~dB}$ higher received signal power than the Vivalde system does for the small metal target and the man, respectively, which states the superiority of the self-grounded BOW-Tie to the Vivalde in this case.

\section{CONCLUSION}

A new small, low-profile and directional UWB antenna the self-grounded Bow-Tie is presented in the paper. Due to its many good characteristics, it can be foreseen that the self-grounded Bow-Tie will find many applications in UWB technology.

The self-grounded Bow-tie antenna is protected by a pending patent [17].

\section{ACKNOWLEDGMENT}

The authors would like to thank Yinan Yu and Sohaib Maalik for their measurements presented in from Fig. 9 to Fig. 12.

\section{REFERENCES}

[1] O. Lodge, "Electric Telegraphy," U.S. Patent 609 154, Aug. 15, 1898. 


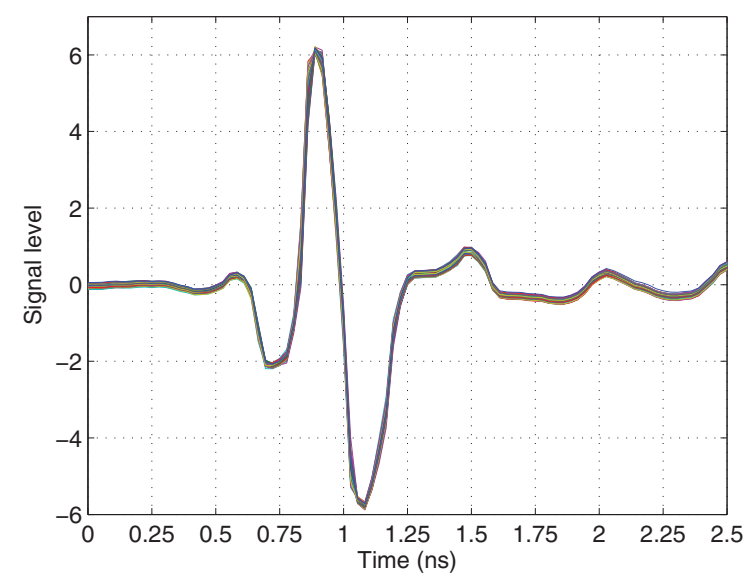

Fig. 9. Time-domain impulse response measurement with the UWB radar system: the two antennas separated by $250 \mathrm{~mm}$ and 50 continuous measurements overlapped.
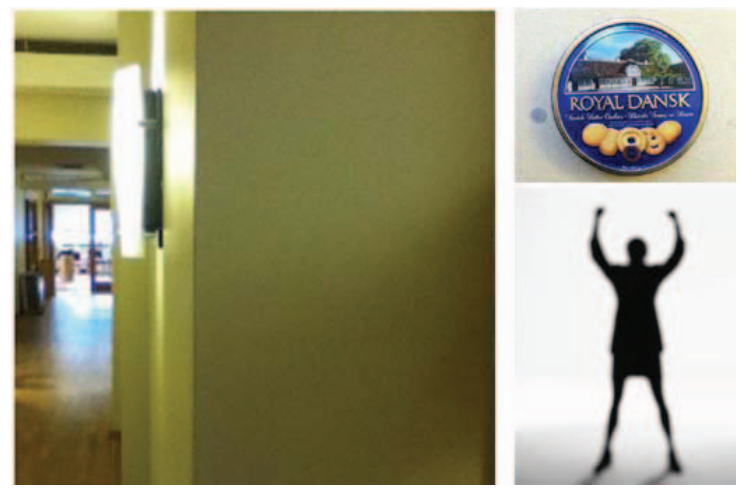

Fig. 10. Penetration test for self-grounded Bow-Tie through a $515 \mathrm{~mm}$ thick concrete wall with a meta box of $213 \mathrm{~mm}$ in diameter (right up) and a man (right down) behind the wall

[2] G. H. Brown and O. M. Woodward, "Experimentally determined radiation characteristics of conical and triangular antennas," RCA Rev., pp. 425-452, Dec. 1952.

[3] P. J. Gibson, "The Vivaldi aerial," Proc. 9th European. Microwave Conference, pp. 101-105, 1979.

[4] ETS-Lindgren horn; http://www.ets-lindgren.com/pdf/3164-05.pdf,

[5] Kenny S. Ryu and Ahmed A. Kishk, "UWB antenna with single or dual band-notches for lower WLAN band and upper WLAN band," IEEE Transa. Antennas Propag., vol. 57, no. 12, pp. 3942-3950, Dec. 2009.

[6] R. Olsson, P.-S. Kildal and S. Weinreb, "The Eleven antenna: a compact low-profile decade bandwidth dual polarized feed for reflector antennas," IEEE Trans. Antennas Propogat., vol. 54, no.2, pp. 368-375, Feb. 2006.

[7] J. Yang, X. Chen, N. Wadefalk, P.-S. Kildal, "Design and realization of a linearly polarized Eleven feed for 1-10 GHz," IEEE Antennas Wireless Propag. Lett., vol. 8, pp. 64-68, 2009..

[8] J. Yang and P.-S. Kildal, "Optimization of large log-periodic dual-dipole antenna by using genetic algorithm on embedded element in small logperiodic array," 3rd Eur. Conf. on Antennas Propagat. (EuCAP2009), Berlin, 23 - 27 March 2009.

[9] J. Yang, D. Nyberg and J. Yin, "Impedance matrix of a folded dipole pair under Eleven configuration," IET Microwaves Antennas Propagat., vol. 4, no. 6, pp. 697-703, June, 2010.

[10] J. Yang, "On Conditions for Constant Radiation Characteristics for Logperiodic Array Antennas," IEEE Trans. on Antennas Propagat., vol. 58,

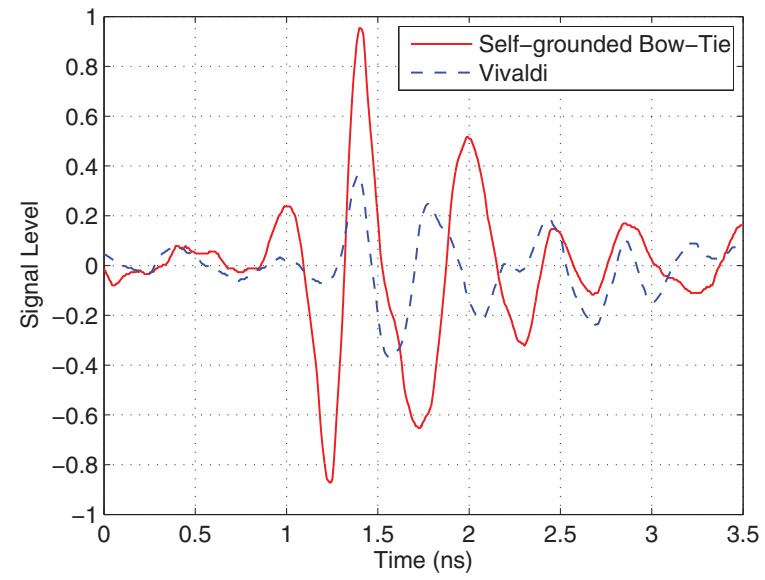

Fig. 11. Received signal for penetration test with a meta box of $213 \mathrm{~mm}$ in diameter as the target behind a $515 \mathrm{~mm}$ thick concrete wall.

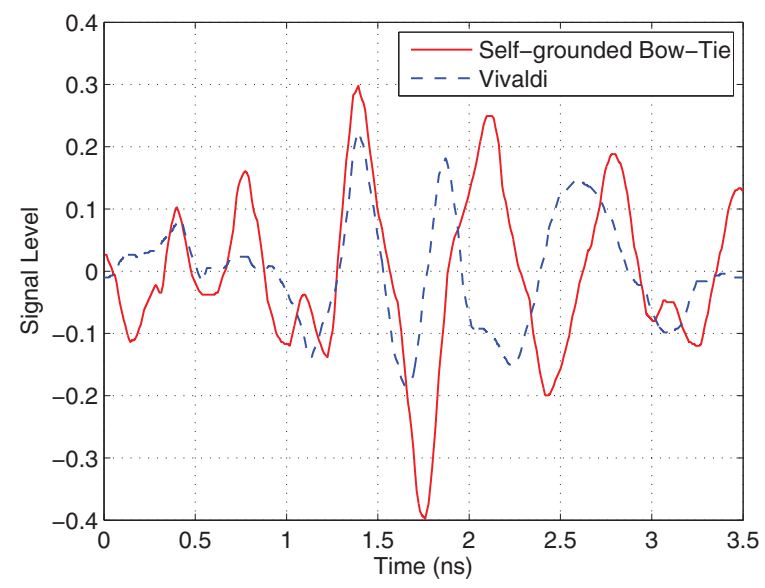

Fig. 12. Received signal for penetration test with a man as the target behind a $515 \mathrm{~mm}$ thick concrete wall.

no. 5, pp. 1521-1526, May, 2010.

[11] J. Yang, P.-S. Kildal, "Optimizing large log-periodic array by computing a small part of it," IEEE Trans. on Antennas Propag. Special Issue on Antennas for Next Generation Radio Telescopes, vol. 59, no. 3, March 2011.

[12] J. Yang, M. Pantaleev, P.-S. Kildal, B. Klein, Y. Karandikar, L. Helldner N. Wadefalk,C. Beaudoin "Cryogenic 2-13 GHz Eleven feed for reflector antennas in future wideband radio telescopes," IEEE Trans. on Antennas Propag. Special Issue on Antennas for Next Generation Radio Telescopes, vol. 59, no. 3, March 2011.

[13] CST Microwave Studio, 3D EM Simulation software, www.cst.com.

[14] D. Nyberg, P.-S. Kildal, J. Carlsson, "Effects of intrinsic radiation Q on mismatch factor of three types of small antennas: single-resonance, gradual-transition and cascaded-resonance types," IET Microwaves Antennas Propagat., vol. 4, no. 1, pp. 83-90, Jan. 2010.

[15] Novelda Nanoscale Impulse Radar: http://www.novelda.no/

[16] W. Wiesbeck, G. Adamiuk, and C. Sturm, "Basic properties and design principles of UWB antennas," Proceedings of the IEEE, vol. 97, no. 2, pp. 372-385, Feb. 2009.

[17] J. Yang and A. Kishk, "Self-grounded Bow-tie antenna," PCT patent application number PCT/SE2010/1001072-6. 